\title{
Affibody molecules as engineered protein drugs
}

\author{
Fredrik Y Frejd ${ }^{1,2}$ and Kyu-Tae $\mathrm{Kim}^{3}$
}

\begin{abstract}
Affibody molecules can be used as tools for molecular recognition in diagnostic and therapeutic applications. There are several preclinical studies reported on diagnostic and therapeutic use of this molecular class of alternative scaffolds, and early clinical evidence is now beginning to accumulate that suggests the Affibody molecules to be efficacious and safe in man. The small size and ease of engineering make Affibody molecules suitable for use in multispecific constructs where AffiMabs is one such that offers the option to potentiate antibodies for use in complex disease.
\end{abstract}

Experimental \& Molecular Medicine (2017) 49, e306; doi:10.1038/emm.2017.35; published online 24 March 2017

\section{INTRODUCTION}

Advances in protein engineering technology in the late 90s and early 2000 allowed the generation of several non-antibody protein scaffold formats. ${ }^{1,2} \mathrm{~A}$ few of the scaffolds have reached early clinical development stage, ${ }^{3}$ and the Affibody molecule class is among those. In this review, recent advances of the Affibody technology will be reviewed with an emphasis on translational research.

In 1997, Nord et al. ${ }^{4}$ demonstrated that a small three-helical subdomain of a natural receptor for the Fc-portion of IgG could be utilized as a general scaffold for diversity. By randomizing amino acids on two of the three helices, large libraries can be constructed, from which potent binders can be isolated by a variety of display methods, ${ }^{5-7}$ and the scaffold has been engineered for improved synthesis and solubility. ${ }^{8}$ Affibody molecules can be selected to a large variety of different proteins, and can be functionalized with genetic fusions to protein modules ${ }^{9,10}$ or by chemical conjugation to toxic molecules, ${ }^{11}$ reviewed in Löfblom et al. ${ }^{12}$

The Affibody molecule is very robust, and has generated useful biotech tools, for example, as the alkali-resistant active component in MabSelect SuRe for affinity purification of mAbs (MabSelect SuRe, Catalog number 17-5438-01, GE Healthcare, Chicago, IL, USA) or as part of a hot start PCR kit, where the Affibody molecule improves the fidelity of the DNA polymerase by binding to it at ambient temperature, and by surviving a large number of heat cycles (Phusion Hot Start II DNA Polymerase, Catalog number: F549L, Thermofisher Scientific, Waltham, MA, USA). The potential for translation to clinical use is being explored and can be categorized in engineered molecules for diagnostic imaging, use for improving therapeutic outcome by providing guidance during surgery, and therapeutically active molecules to be used as drugs.

\section{ENGINEERED AFFIBODY MOLECULES FOR DIAGNOSTIC IMAGING}

Imaging is an important tool to identify, characterize and monitor tumors. There are several metabolic markers that have been developed (the most used one is fluorodeoxyglucosepositron emission tomography (FDG-PET) ${ }^{13}$ ), but a scarcity of tumor receptor-specific tracers. Compared with the other protein based affinity tools (for example, monoclonal antibodies and their fragments), Affibody molecules have very small size and hence have favorable properties for diagnostic imaging. ${ }^{14}$ More specifically, a molecular size below $10 \mathrm{kDa}$ allows more rapid extravasation from blood vessels and penetration into tissue, allowing for rapid reach of tumor targets. ${ }^{15}$ Molecules below $\sim 60 \mathrm{kDa}$ have short plasma half-lives as they are cleared via renal filtration. ${ }^{16}$ Altogether, these properties are favorable for clinical imaging use, allowing for high-contrast images within hours after administration of the tracer.

Affibody molecules specific for tumor associated targets have been generated and characterized preclinically. One of the first targets to be explored was HER2, ${ }^{17}$ and the generation of a high affinity (22 pM) Affibody molecule to HER2 $\left({ }^{7}\right)$ allowed for initial characterization of this molecular class with regards to different valencies and affinities, as well as various nuclidelabeling methods, reviewed in refs 14,18 . Interestingly, it was shown that a monomer Affibody had the same magnitude of tumor uptake as a dimeric higher affinity version, suggesting that small size is of high importance. ${ }^{19,20}$ It was furthermore shown that high affinity (pM KD) is an important feature for

${ }^{1}$ Affibody AB, Solna, Sweden; ${ }^{2}$ Department of Immunology, Genetics and Pathology, Uppsala University, Uppsala, Sweden and ${ }^{3}$ AbClon Inc., Guro-gu, Seoul, Republic of Korea

Correspondence: Dr FY Frejd, Affibody AB, Gunnar Asplunds Allé 24, Solna, SE-171 69, Sweden.

E-mail: fredrik.frejd@affibody.com

Received 7 December 2016; accepted 22 December 2016 
detecting low receptor expression levels but less so for very high expression levels (nм KD is sufficient). ${ }^{21}$

\section{CLINICAL RECEPTOR QUANTIFICATION IN VIVO}

To test the feasibility of targeted detection of tumor HER2 expression in humans, a high affinity HER2-binding Affibody molecule was chemically synthesized and derivatized with 1,4,7,10-tetraazacyclododecane- N,N',N",N"'-tetraacetic acid (DOTA) for complexation of radiometals. The subsequent clinical study demonstrated for the first time that a nonimmunoglobulin derived, non-peptide alternative scaffold protein could target and visualize tumors in humans. ${ }^{22}$ The contrast was very high and allowed tumor detection after injection using single photon computed tomography (SPECT) and an ${ }^{111}$ In-labeled Affibody molecule. The signal was further improved when using positron emission tomography (PET)imaging and ${ }^{68} \mathrm{Ga}$-labeled Affibody, suggesting broad usefulness of the technology for molecular imaging. ${ }^{22}$ To further investigate the clinical utility of HER2 imaging, a larger study was prepared. To facilitate large scale good manufacturing practice (GMP)-production, the Affibody molecule was now produced recombinantly and denoted ABY-025. ${ }^{8,23}$ In a first study, ability to detect HER2 expression as early as $2-3 \mathrm{~h}$ after injection was demonstrated in a small group of patients using ${ }^{111}$ In-labeled Affibody molecule. Brain metastases could be detected as well as metastases near the kidney, the primary excretion organ. ${ }^{24}$ In a second study, GMP-production of ${ }^{68} \mathrm{Ga}$-labeled ABY-025 was established ${ }^{25}$ and investigated for PET-imaging. This methodology allows for quantification of the uptake and allows the use of the Affibody molecule for determination of the HER2 expression levels. Today, the standard biopsy immunohistochemistry Herceptest ${ }^{26}$ is used for characterization of patients, but the drawback is obviously that the information is limited to the one metastasis that could be biopsied. A global imaging quantification would allow for a more precise determination of receptor status in all lesions and thus provide for better guidance of treatment. Indeed, Sörensen et al. ${ }^{27}$ could demonstrate a very good correlation between signal intensity and the control immunohistochemistry score in all metastases tested. Furthermore, conversion from HER2 positive primary tumor to HER2 negative metastases, and vice versa was determined, as well as conversion from HER2 negative tumor to heterogeneous HER2 expression levels in different metastases in the same patient. ${ }^{27,28}$ In 3 of 16 patients, treatment was changed as a direct consequence of the new knowledge of the HER2-status. If the results from these studies are confirmed in later larger trials, ABY-025 may well change the way patients with HER2 positive tumors are treated in the future.

\section{TOWARDS INTRAOPERATIVE IMAGING}

Epidermal growth factor receptor (EGFR) is a protein in the same receptor family as HER2, which is overexpressed in many cancers, especially certain brain cancers. ${ }^{29}$ Like HER2, the receptor is a growth factor receptor, and as overexpression thus promotes tumor growth, ${ }^{30}$ it is in principle difficult for the tumors to escape EGFR targeted therapies. Affibody molecules for EGFR have been generated and as there are quite high levels of EGFR-expression also in normal tissues like skin and liver, binders were isolated that would cross react with murine EGFR to allow for more accurate preclinical studies to be performed. ${ }^{31}$ This has allowed preclinical tumor-targeting studies in mice in presence of endogenous background levels of EGFR, and feasibility of high-contrast tumor images in tumor bearing mice has been demonstrated. ${ }^{32-34}$ Detailed studies on the composition of a peptidic chelator fused to the EGFR-specific Affibody molecule revealed a marked difference in excretion pattern after a change of just a few amino acids. ${ }^{35}$ Inspired by the early tumor-targeting results, Gong et al. ${ }^{36}$ labeled the EGFR-specific Affibody molecule with a near infrared fluorescent probe, to visualize tumors without radioactivity. The EGFR-specific Affibody molecule bound to and was taken up by EGFR-expressing A431 cancer cells in vitro, and in contrast to the natural ligand, EGF, the Affibody molecule did not activate EGFR signaling pathways. When intravenously injected in tumor bearing nude mice, the tumors could be detected as early as one hour after injection, and images of dissected tissue sections confirmed high uptake in the tumor, and liver, both displaying high EGFR-expressing levels, and kidney, the main excreting organ. In combination with a HER2-targeting fluorescently labeled Affibody molecule, it was further shown that the EGFR-specific Affibody molecule could discriminate between EGFR- and HER2-expressing tumors and that the EGFR-specific molecule is a promising candidate for molecular imaging of EGFR-expressing tumors. ${ }^{36}$

The concept has since then been further developed by Pogue and colleagues, ${ }^{37}$ who recognized that a challenge during surgical resection of malignant gliomas is to remove all tumor tissue while sparing as much as possible of the healthy brain tissue surrounding the tumor lesion, to preserve brain function. Today, to better visualize the tumor border, a prodrug is orally administered that is metabolized to a fluorescent dye in the metabolically active cancer cells and surgery is performed using detection of the emitted UV-light. A drawback of UV light is however very short tissue penetration, and that this wavelength is quenched by blood. In addition, it works well only with the most metabolically active lesions. Pogue and colleagues ${ }^{37}$ investigated if one could use the fact that many gliomas have very high expression levels of EGFR. With the hypothesis that a small sized targeting agent would provide better tissue penetration and tumor border contrast than a larger molecule, they compared the EGFR-specific clinically used monoclonal antibody cetuximab ${ }^{38}$ with an EGFR-specific Affibody. Both molecules showed accumulation in orthotopically growing brain tumors in mice. There was however a better contrast in the proliferative tumor border with the smaller Affibody compared with the antibody that seemed to be more restricted to the central part of the tumor where the blood brain barrier was more disrupted $(7 \mathrm{kDa}$ vs $150 \mathrm{kDa})$, and the authors concluded that the Affibody molecule showed the highest potential for use as a clinical imaging tool. ${ }^{37}$ To pave way for a cost efficient potential clinical program, it was demonstrated that even a corresponding microdose of an EGFR-specific 
Affibody molecule, denoted ABY-029, was sufficient for delineation of human glioma xenografts in nude rats. ${ }^{39}$ It was furthermore shown that ABY-029 imaging outperformed the standard 5-aminioleuvulinic acid agent in a direct comparison in orthotopically implanted rats but also that the overall imaging analysis could be further improved if ABY-029 was added to the standard agents and both signals used. ${ }^{40}$ To test the utility of ABY-029 and to address the challenge of translation of novel agents to the clinic with very limited funding, ABY-029 has been synthesized chemically to support a phase 0 clinical trial to obtain initial characterization of the molecule, supported by a single NIH grant. ${ }^{41}$ The first in man study was recently approved by the FDA and ABY-029 will soon be tested in patients with recurrent glioma (NCT02901925).

\section{EARLY STAGE IMAGING AGENTS}

A number of cancer associated targets have been identified for generation of Affibody molecules, and include targets expressed both directly on the cancer cells and targets that are more associated with the physiology of the tumor. HER3 is the third member of the EGFR family described above, and is an important driver of oncogenesis as a heterodimer with other family receptors. ${ }^{42}$ In contrast to EGFR and HER2, HER3 is not massively overexpressed in tumors, and there are substantial levels of normal tissue expression. An Affibody molecule that is cross reactive with the murine target was therefore isolated to study targeting and uptake in relevant models. ${ }^{6,43}$ As the expression levels are relatively low, high affinity variants were needed, and affinity maturation resulted in low picomolar binders ${ }^{44}$ that demonstrated good tumortargeting properties in xenografted mice. ${ }^{45}$ To test the ability to discriminate between high and low HER3 expression, a PET-labeling methodology was established, and it was shown using micro PET that uptake of the tracer was much higher in xenografts with high HER3 expression (BT474, BxPC-3) as compared with the xenografts with low HER3 expression (A431). ${ }^{46}$ To address the heterodimeric nature of HER3 signaling, a novel bispecific format was constructed targeting both HER2 and HER3, and with a half-life extension module in the linker region. The molecule could simultaneously bind both HER2 and HER3 and efficiently blocked ligand-induced phosphorylation, indicating a potential use for therapy. ${ }^{47}$ The molecule is currently being evaluated further for therapeutic applications.

Another target with low tumor expression levels is the IGF1R, making it a challenging candidate for imaging. Tracer requirements are high enough affinity and good enough contrast due to rapid blood clearance to be able to monitor IGF1R, which is reported to be highly important in resistance mechanisms of tumors. Gräslund and colleagues ${ }^{48}$ tested if the Affibody technology could be used and IGF1R binders were isolated. Feasibility of tumor targeting in DU-145 prostate cancer xenografts in mice was shown, ${ }^{21}$ and subsequent studies showed useful contrast between tumor and most other organs. ${ }^{49,50}$
An interesting class of tumor imaging targets relates to markers that are associated with the physiology of the tumor environment, rather than being directly expressed on the tumor cells. Such markers include CAIX that is upregulated in the hypoxic conditions in solid tumors, ${ }^{51}$ VEGFR2 being a driver for tumoral angiogenesis, ${ }^{52}$ and PDGFR, that is expressed on activated pericytes and fibroblasts in tumor stroma. ${ }^{53}$ Imaging tracers for such markers could provide a more general imaging modality that in principle would be less restricted to tumor cell phenotype. Affibody molecules have been isolated and shown to be able to target tumors having expression of CAIX ${ }^{33,54}$ and PDGFR. ${ }^{55-57}$ As expression levels of VEGFR2 are low, and available only in a small cell population within the tumor, efforts have been directed towards enhancing the affinity of the VEGFR2-specific Affibody molecule. Here, two Affibody molecules binding to two distinctly different target epitopes were combined, and a biparatopic format was engineered, with substantially improved binding affinity. ${ }^{58}$ In addition, the resulting biparatopic molecule blocked ligand receptor interaction and inhibited angiogenic sprout formation of endothelial cells as efficiently as the antibody ramucirumab, which is approved for treatment of metastasized lung, colorectal and gastric cancer. ${ }^{59}$ This suggests that Affibody molecules could be used for therapeutic intervention, and will be discussed below.

\section{TOWARDS THERAPEUTIC APPLICATION OF AFFIBODY MOLECULES}

Affibody molecules have the ability to bind protein targets with high affinity and selectivity. In contrast to antibodies that have $\mathrm{Fc}$, however, they lack half-life extension and effector function modules. Therapeutic action can thus either be directly carried out by blocking ligand receptor interactions, as shown by antibodies in inflammatory conditions, ${ }^{60,61}$ or by functionalizing the Affibody molecules to have long half-lives and toxic payloads. One of the first therapy examples using this approach was the use of a HER2-targeted Affibody molecule, ${ }^{7}$ which had shown excellent tumor uptake in mice, ${ }^{62}$ and is in development for metastasized breast cancer. ${ }^{24,27}$ The diagnostic agent however, had relatively high kidney uptake levels and could not be directly used for targeting of potent toxic payloads. Kidney uptake of small proteins is a normal physiologic mechanism, and the size cut off is around $60 \mathrm{kDa}$, just below the size of serum albumin, which is a major plasma protein with very long half-life. ${ }^{63}$ To solve the problem of kidney uptake, a small engineered albumin-binding domain (ABD) was genetically fused to the HER2-specific Affibody molecule. The resulting fusion protein indeed displayed very long circulatory half-life with increased dose to the tumor, substantially reduced kidney uptake and demonstrated potent antitumor effect in a micrometastatic model of breast cancer when conjugated with the therapeutic radionuclide ${ }^{177} \mathrm{Lu}^{9}{ }^{9}$ The construct was further improved using an affinity matured ABD with femtomolar-binding affinity, ${ }^{64}$ an optimized HER2binding Affibody molecule, ${ }^{8}$ and site-specific labeling of the radionuclide by using a cysteine as chemical handle, further 
lowering the kidney uptake and increasing tumor to normal organ ratios. ${ }^{9}$ The albumin-binding domain has subsequently been optimized and deimmunized, ${ }^{65}$ and has been demonstrated to enhance the half-life in several different species ${ }^{66}$ and recently also in humans (NCT02690142).

Radionuclide payloads have several advantages, but also some drawbacks in terms of logistics and some potential side effects. Other payloads have been investigated with Affibody molecules, and an attractive approach is to use a protein toxin, since this allows for a manufacturing process based on a single polypeptide chain. Pseudomonas exotoxin is a well characterized toxin that in a truncated version (PE38) has been fused to antibody fragments to create a targeted immunotoxin. This was tested also for the HER2-targeting Affibody molecule, ${ }^{67}$ and the resulting fusion protein was shown to completely eradicate large BT474 tumors in xenograft models. ${ }^{10}$ The immunotoxin had a short half-life to minimize potential side effects, but speculating that longer half-life would enhance the effect of the construct, Graslund and co-workers ${ }^{68}$ constructed a triple functional targeted toxin by fusing a deimmunized PE38 to the albumin-binding domain described above and to the targeting HER2 Affibody molecule. The fusion protein reacted with serum albumin, and IC50s from 6 to $300 \mathrm{pm}$ were reported in cell assays, where a short incubation of $10 \mathrm{~min}$ resulted in substantial cell killing. Also Guo et al. ${ }^{69}$ demonstrated the ability to fuse PE38 to ABD and HER2 to enhance potency, and they found that in comparison to immunotoxin without $\mathrm{ABD}$, the half-life was improved almost 25 fold, and with improved antitumor effects in NCI-N87 tumor xenografts.

A general problem when using small toxic payload molecules conjugated to carrier proteins is the toxic side effects that occurs where the drug is degraded or, with radioactive payloads, in the bone marrow due to high blood exposure. Pre-targeting is a way to solve this problem by separating the targeting moiety and the effector domain in time. This is especially attractive with the Affibody molecules as they have both good tumor targeting and rapid kinetics. ${ }^{14,27}$ In principle, the two step targeting approach could be carried out within a day, which substantially would improve future clinical routine. In a first test, Tolmachev and colleagues ${ }^{70}$ tested an anti-HER2 Affibody-based peptide nucleic acid with a complementary hybridization probe that showed low kidney uptake and specific high affinity binding on HER2-expressing cells in vitro with substantial tumor uptake in HER2-expressing xenografts in vivo. The uptake was specific and tumor to blood levels of 50:1 and tumor to kidney of 2:1 suggests potential for radioimmunotherapy. ${ }^{54}$ In a second study, trans-cyclooctene (TCO) and tetrazine were tested as a system to enhance the accumulation of radiometals in tumor xenografts over kidneys. TCO was conjugated to a HER2-targeting Affibody molecule that was shown to bind to HER2-expressing cells. A DOTAderivatized tetrazine was then labeled with radiometals and shown to localize in tumors that were pretargeted with the Affibody-TCO construct. Compared with a directly labeled tracer, pre-targeting resulted in a 56-fold reduction of renal uptake with two times more activity in the tumor as compared with the kidney and 50-fold more activity in the tumor as compared to blood. ${ }^{71}$ In summary, both the peptide nucleic acid and the biorthogonal chemistry approaches allowed administration of the effector module already $4 \mathrm{~h}$ after injection of the targeting agent, and pre-targeting using Affibody molecules with rapid kinetics could be an attractive approach towards more efficient payload therapy.

Blocking of a disease causing agent by binding to it is a tractable therapeutic modality, as it does not require modification of the binding molecule. Monoclonal antibodies have demonstrated the feasibility in several inflammatory conditions, but in some diseases with very high target concentrations there is a limitation in the amount of antibodies that can be delivered in the one milliliter volume that can be administered subcutaneously without discomfort. Typically, very high antibody concentrations are needed $\left(>100 \mathrm{mg} \mathrm{ml}^{-1}\right)$, and if that is not sufficient, intravenous infusions are required, which means hospital visits and limitations for patients living in remote areas. Here, the Affibody technology, which given the small molecular size offers the potential to increase the dose 10-fold in the same volume, would be attractive to employ. In several rare autoimmune diseases, the complement system is aberrantly activated. One central part of the complement system is C5, and eculizumab is a C5 blocking monoclonal antibody approved for the treatment of paroxysmal nocturnal hemoglobinuria $(\mathrm{PNH})$ and atypical hemolytic uremic syndrome (aHUS), two rare disease caused by an autoimmune activation. ${ }^{72,73}$ The drug is dosed by infusion at very high doses, up to $1200 \mathrm{mg}$ every second week. An alternative with potential for subcutaneous home use administration and with less costly production would be attractive. Affibody molecules specific for C5 were generated using phage display technology, and candidates that demonstrated cross reactivity with both rodent and cyno and that had affinity in the low to subnanomolar range were selected. The molecules were engineered in different formats for tailored kinetic properties, including PEGylation, Fc-fusion or fusion to ABD for albumin binding. An ABD-fused anti-C5 Affibody molecule that could inhibit C5-dependent hemolysis in vitro and potently block C5 in vivo in a Zymosan-induced peritonitis mouse model was recently tested in healthy volunteers (NCT02083666). ${ }^{74}$

Another target where very high antibody doses are used in clinical trials is Abeta in Alzheimer's disease (AD). Several antibodies employed in current $\mathrm{AD}$ clinical trials are administered at very high doses corresponding to $300-800 \mathrm{mg}$ per patient $(80 \mathrm{~kg})$, which requires intravenous infusion every three weeks or so. ${ }^{75,76}$ Given the notion of a life-long preventive treatment and the logistics of the high number of potential AD patients that would thus require dosing, it would be highly desirable to identify a drug that could be administered at home in an outpatient subcutaneous setting instead of requiring i.v. infusion centers. Affibody molecules specific for Abeta were developed to address this challenge. Abeta is a key component in the development of $\mathrm{AD}$, and is present in several different isoforms. ${ }^{77}$ It is believed that inhibition of the ability of Abeta 


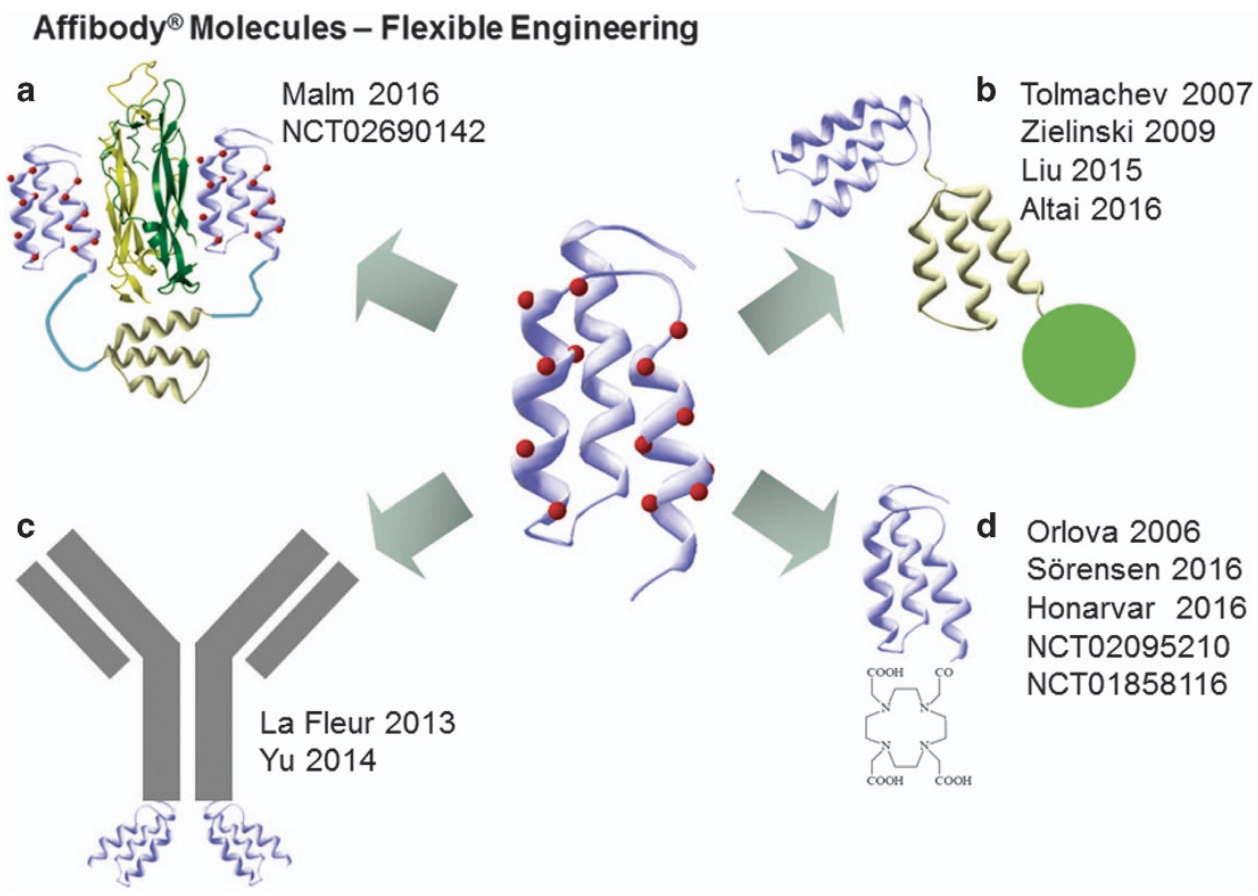

Figure 1 Affibody molecules can be engineered in different formats. (a) Hetero- or homospecific bivalent and half-life extended, (b) monospecific half-life extended with payload, (c) as additional molecular specifier in multispecific antibody based formats and (d) as monospecific carrier of a payload with rapid kinetics and high contrast.

to aggregate to form plaques could be a major therapeutic opportunity. Stahl and colleagues ${ }^{5}$ isolated an initial set of Abeta binding Affibody molecules, that preferentially bound non-aggregated Abeta. The protein structure was solved using NMR and it was shown that the Affibody molecule ZAbeta3 stabilized a $\beta$-hairpin of the monomeric amyloid- $\beta$ peptide to act as an inhibitor of $A \beta$ fibrillation. ${ }^{78}$ To test if the ZAbeta3 Affibody molecule could act therapeutically in vivo, it was tested in a fruit fly model of AD. ZAbeta3 in monomeric and dimeric formats were co-expressed in the brain of fruit flies expressing either Abeta 42 or the aggressive variant E22G of Abeta and the life span of the fruit flies was studied. The results were impressive, with completely abolished neurotoxic effects in the flies that received the dimeric Affibody molecule. ${ }^{79}$ To further improve this molecule, it was hypothesized that enhanced affinity was needed. A Staphylococcal bacterial display system was established to thoroughly characterize the Affibody molecules. In addition to functional expression, determination of affinity and $\mathrm{pH}$-sensitivity, effects from truncation of the N-terminal part of the Affibody molecule was investigated $^{80}$ On the basis of this work and structure information available, ${ }^{78}$ an affinity maturation effort was undertaken using Staphylococcal display to guide selection of improved binders. Two different affinity maturation libraries were designed and subjected to selections. Improved binders could be isolated and the best had a 50-fold improved affinity with around 300 pм KD. ${ }^{81}$ This binder was fused to an ABD and tested in vivo in a double transgenic mouse model of $\mathrm{AD}$ and shown to efficiently protect the mice from Abeta-induced pathology. ${ }^{81}$
The clinically most advanced Affibody molecule is currently an engineered IL-17-specific ligand trap. IL-17 is a key driving molecule in psoriasis, and moderate to severe psoriasis strongly impacts the quality of patient lives. ${ }^{82}$ To create an IL-17 blocking molecule with a potency superior to the monoclonal antibodies ixekizumab ${ }^{83}$ and secukinumab, ${ }^{84}$ IL-17-specific Affibody molecules were formatted into a small $18 \mathrm{kDa}$ dimeric construct with built in long plasma half-life using a previously reported format. ${ }^{47}$ By simultaneously binding and blocking both subunits of the dimeric IL-17 molecule, the affinity was increased ten-thousand fold to sub-picomolar KD affinity. No adverse findings were reported in preclinical toxicity studies, and the molecule was named ABY-035 and has entered clinical development. Recently, the healthy volunteer dose escalation part of the phase I study was completed and initial results in more than 50 subjects suggest the compound to be safe and well tolerated (NCT02690142). ABY-035 is being evaluated in patients with plaque psoriasis.

\section{AFFIMABS}

A recent trend in biologics development is towards creation of multispecific therapeutic constructs, with antibodies leading the way. ${ }^{85} \mathrm{~A}$ novel class is using antibodies as a basic scaffold, which is then fused to peptides or alternative scaffolds to functionalize the antibody with enhanced properties. This has been shown with small peptides ${ }^{86}$ and recently also with alternative scaffold proteins. ${ }^{87,88}$ Affibody molecules have been demonstrated to be useful as molecular specifiers for antibodies in several labs, including our own, and more than six different Affibody molecules have been combinatorially fused with 
antibodies to form functional multispecific proteins called 'AffiMabs' ${ }^{87,89}$ There are various formats of multispecific antibodies described which have quite different structures from the canonical IgG format. In contrast, AffiMabs retains symmetric bi-valency and Fc of common IgGs, and AffiMabs are supposed to have corresponding substantial half-life and stability in vivo and facile manufacturability. La Fleur and colleagues showed that it is possible to create pentaspecific antibody constructs using Affibody molecules. They also showed superior in vivo therapeutic activity in a xenograft tumor model when administering a bispecific molecule based on the EGFR-specific antibody cetuximab and a HER3-specific Affibody molecule. The bispecific molecule more efficiently inhibited cell growth of the pancreatic cell line BxPC-3 in vitro and in vivo as compared with the parental cetuximab antibody, which is widely used in cancer therapy. ${ }^{42,87}$

Nygren and co-workers later investigated if AffiMabs could be employed to target inflammatory disease and soluble disease mediators. They noted that many inflammatory conditions are driven by multiple pathways, and reasoned that it would make sense to block at least two of them simultaneously. ${ }^{90-92}$ TNF and IL-6 blocking agents are both approved in RA and with similar efficacy (ADACT trial, NCT01119859). It is believed that IL-6 is more important in the systemic part of the disease whereas TNF controls local inflammation. ${ }^{93}$ To address this hypothesis, an IL-6-specific Affibody molecule that is active for blocking IL-6 trans-signaling was isolated. This is important since there is a lot of IL- 6 bound to shredded IL-6R in the circulation, which can stimulate any cell (trans) that express the co-receptor gp130, ${ }^{94}$ as compared with the action of IL- 6 itself, which can only active cells expressing the receptor on their cell membrane (cis). The Affibody molecule was fused to $\mathrm{N}$ - or C-terminal positions of the heavy or light chain, respectively, of a TNF-specific antibody scaffold derived from adalimumab and characterized for binding and activity. The Affibody molecule was active as fused to all different positions of the antibody, and both parental molecules retained their activity in binding assays. Furthermore, in a growth stimulation TF-1 cell assay, it was shown that the AffiMab was superior in blocking cell activation in comparison with the parental antibody. This was later confirmed in vivo, where TNF and IL-6 was used to activate the production of serum amyloid alpha (SAA) in mice. There was pronounced production of SAA in control mice and only partial inhibition using the TNF-specific antibody, in contrast to the AffiMab, where there was robust and complete inhibition of the cytokine induced SAA response. ${ }^{89}$ This suggests that AffiMabs could become useful in diseases where multiple pathways are activated, and further investigation is warranted. The IL-6/TNF-specific AffiMab is currently in preclinical development (http://www.abclon.com).

\section{CONCLUSION}

In conclusion, Affibody molecules can be used as tools for molecular recognition in diagnostic and therapeutic applications. There are several preclinical studies reported on diagnostic and therapeutic use of this molecular class of alternative scaffolds, and early clinical evidence is now beginning to accumulate that suggests the Affibody molecules to be efficacious and safe in man. The small size and ease of engineering make Affibody molecules suitable for use in multispecific constructs (Figure 1) where AffiMabs is one such that offers the option to potentiate antibodies for use in complex disease.

\section{CONFLICT OF INTEREST}

The authors declare no conflict of interest.

1 Binz HK, Amstutz P, Pluckthun A. Engineering novel binding proteins from nonimmunoglobulin domains. Nat Biotechnol 2005; 23: 1257-1268.

2 Lofblom J, Frejd FY, Stahl S. Non-immunoglobulin based protein scaffolds. Curr Opin Biotechnol 2011; 22: 843-848.

3 Vazquez-Lombardi R, Phan TG, Zimmermann C, Lowe D, Jermutus L, Christ D. Challenges and opportunities for non-antibody scaffold drugs. Drug Discov Today 2015; 20: 1271-1283.

4 Nord K, Gunneriusson E, Ringdahl J, Stahl S, Uhlen M, Nygren PA. Binding proteins selected from combinatorial libraries of an alpha-helical bacterial receptor domain. Nat Biotechnol 1997; 15: 772-777.

5 Gronwall C, Jonsson A, Lindstrom S, Gunneriusson E, Stahl S, Herne N. Selection and characterization of Affibody ligands binding to Alzheimer amyloid beta peptides. J Biotechnol 2007; 128: 162-183.

6 Kronqvist N, Malm M, Gostring L, Gunneriusson E, Nilsson M, Hoiden Guthenberg I et al. Combining phage and staphylococcal surface display for generation of ErbB3-specific Affibody molecules. Protein Eng Des Sel 2011; 24: 385-396.

7 Orlova A, Magnusson M, Eriksson TL, Nilsson M, Larsson B, Hoiden-Guthenberg $\mathrm{I}$ et al. Tumor imaging using a picomolar affinity HER2 binding affibody molecule. Cancer Res 2006; 66: 4339-4348.

8 Feldwisch J, Tolmachev V, Lendel C, Herne N, Sjoberg A, Larsson B et al. Design of an optimized scaffold for affibody molecules. J Mol Biol 2010; 398: 232-247.

9 Tolmachev V, Orlova A, Pehrson R, Galli J, Baastrup B, Andersson K et al. Radionuclide therapy of HER2-positive microxenografts using a 177Lu-labeled HER2-specific Affibody molecule. Cancer Res 2007; 67: 2773-2782.

10 Zielinski R, Lyakhov I, Hassan M, Kuban M, Shafer-Weaver K, Gandjbakhche A et al. HER2-affitoxin: a potent therapeutic agent for the treatment of HER2-overexpressing tumors. Clin Cancer Res 2011; 17: 5071-5081.

11 Altai M, Liu H, Orlova A, Tolmachev V, Graslund T. Influence of molecular design on biodistribution and targeting properties of an Affibody-fused HER2-recognising anticancer toxin. Int J Oncol 2016; 49: 1185-1194.

12 Lofblom J, Feldwisch J, Tolmachev V, Carlsson J, Stahl S, Frejd FY. Affibody molecules: engineered proteins for therapeutic, diagnostic and biotechnological applications. FEBS Lett 2010; 584: 2670-2680.

13 Som P, Atkins HL, Bandoypadhyay D, Fowler JS, MacGregor RR, Matsui K et al. A fluorinated glucose analog, 2-fluoro-2-deoxy-D-glucose (F-18): nontoxic tracer for rapid tumor detection. J Nucl Med 1980; 21: 670-675.

14 Feldwisch J, Tolmachev V. Engineering of affibody molecules for therapy and diagnostics. Methods Mol Biol 2012; 899: 103-126.

15 Schmidt MM, Wittrup KD. A modeling analysis of the effects of molecular size and binding affinity on tumor targeting. Mol Cancer Ther 2009; 8: 2861-2871.

16 Wittrup KD, Thurber GM, Schmidt MM, Rhoden JJ. Practical theoretic guidance for the design of tumor-targeting agents. Methods Enzymol 2012; 503: 255-268.

17 Holbro T, Beerli RR, Maurer F, Koziczak M, Barbas CF 3rd, Hynes NE. The ErbB2/ErbB3 heterodimer functions as an oncogenic unit: ErbB2 requires ErbB3 to drive breast tumor cell proliferation. Proc Natl Acad Sci USA 2003; 100: 8933-8938.

18 Nilsson FY, Tolmachev V. Affibody molecules: new protein domains for molecular imaging and targeted tumor therapy. Curr Opin Drug Discov Devel 2007; 10: 167-175. 
19 Orlova A, Wallberg H, Stone-Elander S, Tolmachev V. On the selection of a tracer for PET imaging of HER2-expressing tumors: direct comparison of a 124 l-labeled affibody molecule and trastuzumab in a murine xenograft model. J Nucl Med 2009; 50: 417-425.

20 Tolmachev V, Mume E, Sjoberg S, Frejd FY, Orlova A. Influence of valency and labelling chemistry on in vivo targeting using radioiodinated HER2-binding Affibody molecules. Eur J Nucl Med Mol Imaging 2009; 36 : 692-701.

21 Tolmachev V, Tran TA, Rosik D, Sjoberg A, Abrahmsen L, Orlova A. Tumor targeting using affibody molecules: interplay of affinity, target expression level, and binding site composition. J Nucl Med 2012; 53: 953-960.

22 Baum RP, Prasad V, Muller D, Schuchardt C, Orlova A, Wennborg A et al. Molecular imaging of HER2-expressing malignant tumors in breast cancer patients using synthetic $111 \mathrm{In}$ - or 68Ga-labeled affibody molecules. J Nucl Med 2010; 51: 892-897.

23 Ahlgren S, Orlova A, Wallberg H, Hansson M, Sandstrom M, Lewsley R et al. Targeting of HER2-expressing tumors using $111 \mathrm{ln}-\mathrm{ABY}-025$, a second-generation affibody molecule with a fundamentally reengineered scaffold. J Nucl Med 2010; 51: 1131-1138.

24 Sorensen J, Sandberg D, Sandstrom M, Wennborg A, Feldwisch J, Tolmachev $V$ et al. First-in-human molecular imaging of HER2 expression in breast cancer metastases using the $111 \mathrm{In}-\mathrm{ABY}-025$ affibody molecule. J Nucl Med 2014; 55: 730-735.

25 Velikyan I, Wennborg A, Feldwisch J, Lindman H, Carlsson J, Sorensen J. Good manufacturing practice production of [(68)Ga]Ga-ABY-025 for HER2 specific breast cancer imaging. Am J Nucl Med Mol Imaging 2016; 6: 135-153.

26 Pauletti G, Dandekar S, Rong H, Ramos L, Peng H, Seshadri R et al. Assessment of methods for tissue-based detection of the HER-2/neu alteration in human breast cancer: a direct comparison of fluorescence in situ hybridization and immunohistochemistry. J Clin Oncol 2000; 18: 3651-3664.

27 Sorensen J, Velikyan I, Sandberg D, Wennborg A, Feldwisch J, Tolmachev V et al. Measuring HER2-receptor expression in metastatic breast cancer using [68Ga]ABY-025 Affibody PET/CT. Theranostics 2016; 6: 262-271.

28 Sandstrom M, Lindskog K, Velikyan I, Wennborg A, Feldwisch J, Sandberg $D$ et al. Biodistribution and radiation dosimetry of the antiHER2 Affibody molecule 68Ga-ABY-025 in breast cancer patients. J Nucl Med 2016; 57: 867-871.

29 Libermann TA, Nusbaum HR, Razon N, Kris R, Lax I, Soreq H et al. Amplification, enhanced expression and possible rearrangement of EGF receptor gene in primary human brain tumours of glial origin. Nature 1985; 313: 144-147.

30 Yarden Y, Sliwkowski MX. Untangling the ErbB signalling network. Nat Rev Mol Cell Biol 2001; 2: 127-137.

31 Friedman M, Orlova A, Johansson E, Eriksson TL, Hoiden-Guthenberg I, Tolmachev $\mathrm{V}$ et al. Directed evolution to low nanomolar affinity of a tumor-targeting epidermal growth factor receptor-binding affibody molecule. J Mol Biol 2008; 376: 1388-1402.

32 Andersson KG, Oroujeni M, Garousi J, Mitran B, Stahl S, Orlova A et al. Feasibility of imaging of epidermal growth factor receptor expression with ZEGFR:2377 affibody molecule labeled with 99mTc using a peptide-based cysteine-containing chelator. Int J Oncol 2016; 49: 2285-2293.

33 Garousi J, Honarvar H, Andersson KG, Mitran B, Orlova A, Buijs J et al. Comparative evaluation of Affibody molecules for radionuclide imaging of in vivo expression of carbonic anhydrase IX. Mol Pharm 2016; 13 : 3676-3687.

34 Tolmachev V, Rosik D, Wallberg H, Sjoberg A, Sandstrom M, Hansson M et al. Imaging of EGFR expression in murine xenografts using site-specifically labelled anti-EGFR 111In-DOTA-Z EGFR:2377 Affibody molecule: aspect of the injected tracer amount. Eur J Nucl Med Mol Imaging 2010; 37: 613-622.

35 Hofstrom C, Altai M, Honarvar H, Strand J, Malmberg J, Hosseinimehr SJ et al. HAHAHA, HEHEHE, HIHIHI, or HKHKHK: influence of position and composition of histidine containing tags on biodistribution of [(99m)Tc(CO) 3](+)-labeled affibody molecules. J Med Chem 2013; 56: 4966-4974.

36 Gong H, Kovar J, Little G, Chen H, Olive DM. In vivo imaging of xenograft tumors using an epidermal growth factor receptor-specific affibody molecule labeled with a near-infrared fluorophore. Neoplasia 2010; 12 : 139-149.

37 Sexton K, Tichauer K, Samkoe KS, Gunn J, Hoopes PJ, Pogue BW. Fluorescent affibody peptide penetration in glioma margin is superior to full antibody. PLOS ONE 2013; 8: e60390.
38 Jonker DJ, O'Callaghan CJ, Karapetis CS, Zalcberg JR, Tu D, Au HJ et al. Cetuximab for the treatment of colorectal cancer. N Engl J Med 2007; 357: 2040-2048.

39 deSouza AL, Marra K, Gunn J, Samkoe KS, Hoopes PJ, Feldwisch J et al. Fluorescent Affibody molecule administered in vivo at a microdose level labels EGFR expressing glioma tumor regions. Mol Imaging Biol 2017; 19: 41-48.

40 Elliott JT, Marra K, Evans LT, Davis SC, Samkoe KS, Feldwisch J et al. Simultaneous in vivo fluorescent markers for perfusion, protoporphyrin metabolism and EGFR expression for optically guided identification of orthotopic glioma. Clin Cancer Res (e-pub ahead of print 14 February 2017; doi:10.1158/1078-0432.CCR-16-1400).

41 Samkoe KS, Gunn JR, Marra K, Hull SM, Moodie KL, Feldwisch J et al. Toxicity and pharmacokinetic profile for single-dose injection of ABY-029: a fluorescent anti-EGFR synthetic Affibody molecule for human use. Mol Imaging Biol (e-pub ahead of print 1 December 2016; doi:10.1007/ s11307-016-1033-y).

42 Malm M, Frejd FY, Stahl S, Lofblom J. Targeting HER3 using monoand bispecific antibodies or alternative scaffolds. MAbs 2016; 8: 1195-1209.

43 Gostring L, Malm M, Hoiden-Guthenberg I, Frejd FY, Stahl S, Lofblom J et al. Cellular effects of HER3-specific affibody molecules. PLOS ONE 2012; 7: e40023.

44 Malm M, Kronqvist N, Lindberg H, Gudmundsdotter L, Bass T, Frejd FY et al. Inhibiting HER3-mediated tumor cell growth with affibody molecules engineered to low picomolar affinity by position-directed error-prone PCR-like diversification. PLOS ONE 2013; 8: e62791.

45 Orlova A, Malm M, Rosestedt M, Varasteh Z, Andersson K, Selvaraju RK et al. Imaging of HER3-expressing xenografts in mice using a $(99 \mathrm{~m}) \mathrm{Tc}(\mathrm{CO})$ 3-HEHEHE-Z HER3:08699 affibody molecule. Eur J Nucl Med Mol Imaging 2014; 41: 1450-1459.

46 Rosestedt M, Andersson KG, Mitran B, Tolmachev V, Lofblom J, Orlova A et al. Affibody-mediated PET imaging of HER3 expression in malignant tumours. Sci Rep 2015; 5: 15226.

47 Malm M, Bass T, Gudmundsdotter L, Lord M, Frejd FY, Stahl S et al. Engineering of a bispecific affibody molecule towards HER2 and HER3 by addition of an albumin-binding domain allows for affinity purification and in vivo half-life extension. Biotechnol J 2014; 9: $1215-1222$.

48 Li J, Lundberg E, Vernet E, Larsson B, Hoiden-Guthenberg I, Graslund T. Selection of affibody molecules to the ligand-binding site of the insulin-like growth factor-1 receptor. Biotechnol App/ Biochem 2010; 55: 99-109.

49 Mitran B, Altai M, Hofstrom C, Honarvar H, Sandstrom M, Orlova A et al. Evaluation of 99mTc-Z IGF1R:4551-GGGC affibody molecule, a new probe for imaging of insulin-like growth factor type 1 receptor expression. Amino Acids 2015; 47: 303-315.

50 Orlova A, Hofstrom C, Strand J, Varasteh Z, Sandstrom M, Andersson K et al. [99mTc(CO)3]+-(HE)3-ZIGF1R:4551, a new Affibody conjugate for visualization of insulin-like growth factor-1 receptor expression in malignant tumours. Eur J Nucl Med Mol Imaging 2013; 40: 439-449.

51 Pastorek J, Pastorekova S. Hypoxia-induced carbonic anhydrase IX as a target for cancer therapy: from biology to clinical use. Semin Cancer Biol 2015; 31: 52-64.

52 Tugues S, Koch S, Gualandi L, Li X, Claesson-Welsh L. Vascular endothelial growth factors and receptors: anti-angiogenic therapy in the treatment of cancer. Mol Aspects Med 2011; 32: 88-111.

53 Paulsson J, Ehnman M, Ostman A. PDGF receptors in tumor biology: prognostic and predictive potential. Future Oncol 2014; 10: 1695-1708.

54 Honarvar H, Westerlund K, Altai M, Sandstrom M, Orlova A, Tolmachev V et al. Feasibility of Affibody molecule-based PNA-mediated radionuclide pretargeting of malignant tumors. Theranostics 2016; 6: 93-103.

55 Lindborg M, Cortez E, Hoiden-Guthenberg I, Gunneriusson E, von Hage E, Syud $\mathrm{F}$ et al. Engineered high-affinity affibody molecules targeting plateletderived growth factor receptor beta in vivo. J Mol Biol 2011; 407: 298-315.

56 Strand J, Varasteh Z, Eriksson O, Abrahmsen L, Orlova A, Tolmachev V. Gallium-68-labeled affibody molecule for PET imaging of PDGFRbeta expression in vivo. Mol Pharm 2014; 11: 3957-3964.

57 Tolmachev V, Varasteh Z, Honarvar H, Hosseinimehr SJ, Eriksson O, Jonasson $\mathrm{P}$ et al. Imaging of platelet-derived growth factor receptor beta expression in glioblastoma xenografts using affibody molecule 111In-DOTA-Z09591. J Nucl Med 2014; 55: 294-300. 
58 Fleetwood F, Klint S, Hanze M, Gunneriusson E, Frejd FY, Stahl S et al. Simultaneous targeting of two ligand-binding sites on VEGFR2 using biparatopic Affibody molecules results in dramatically improved affinity. Sci Rep 2014; 4: 7518.

59 Fleetwood F, Guler R, Gordon E, Stahl S, Claesson-Welsh L, Lofblom J. Novel affinity binders for neutralization of vascular endothelial growth factor (VEGF) signaling. Cell Mol Life Sci 2016; 73: 1671-1683.

60 Campa M, Menter A. A review of emerging IL-17 inhibitors in the treatment of psoriasis focusing on preclinical through phase II studies. Expert Opin Investig Drugs 2016; 25: 1337-1344.

61 Smolen JS, Burmester GR, Combe B, Curtis JR, Hall S, Haraoui B et al. Head-to-head comparison of certolizumab pegol versus adalimumab in rheumatoid arthritis: 2-year efficacy and safety results from the randomised EXXELERATE study. Lancet 2016; 388: 2763-2774.

62 Tolmachev V, Nilsson FY, Widstrom C, Andersson K, Rosik D, Gedda L et al. 111In-benzyl-DTPA-ZHER2:342, an affibody-based conjugate for in vivo imaging of HER2 expression in malignant tumors. J NuCl Med 2006; 47: 846-853.

63 Peters T Jr. Serum albumin. Adv Protein Chem 1985; 37: 161-245.

64 Jonsson A, Dogan J, Herne N, Abrahmsen L, Nygren PA. Engineering of a femtomolar affinity binding protein to human serum albumin. Protein Eng Des Sel 2008; 21: 515-527.

65 Zurdo J, Arnell A, Obrezanova O, Smith N, Gomez de la Cuesta R, Gallagher TR et al. Early implementation of $\mathrm{QbD}$ in biopharmaceutical development: a practical example. Biomed Res Int 2015; 2015: 605427.

66 Levy OE, Jodka CM, Ren SS, Mamedova L, Sharma A, Samant M et al. Novel exenatide analogs with peptidic albumin binding domains: potent anti-diabetic agents with extended duration of action. PLOS ONE 2014; 9: e87704.

67 Zielinski R, Lyakhov I, Jacobs A, Chertov O, Kramer-Marek G, Francella N et al. Affitoxin-a novel recombinant, HER2-specific, anticancer agent for targeted therapy of HER2-positive tumors. J Immunother 2009; 32: 817-825.

68 Liu H, Seijsing J, Frejd FY, Tolmachev V, Graslund T. Target-specific cytotoxic effects on HER2-expressing cells by the tripartite fusion toxin ZHER2:2891-ABD-PE38X8, including a targeting affibody molecule and a half-life extension domain. Int J Oncol 2015; 47: 601-609.

69 Guo R, Guo W, Cao L, Liu H, Liu J, Xu H et al. Fusion of an albumin-binding domain extends the half-life of immunotoxins. Int J Pharm 2016; 511: 538-549.

70 Westerlund K, Honarvar H, Tolmachev V, Eriksson Karlstrom A. Design, preparation, and characterization of PNA-based hybridization probes for affibody-molecule-mediated pretargeting. Bioconjug Chem 2015; 26 : 1724-1736.

71 Altai M, Perols A, Tsourma M, Mitran B, Honarvar H, Robillard M et al. Feasibility of Affibody-based bioorthogonal chemistry-mediated radionuclide pretargeting. J Nucl Med 2016; 57: 431-436.

72 Garjau M, Azancot M, Ramos R, Sanchez-Corral P, Montero MA, Seron D. Early treatment with eculizumab in atypical haemolytic uraemic syndrome. Clin Kidney J 2012; 5: 31-33.

73 Hillmen P, Young NS, Schubert J, Brodsky RA, Socie G, Muus P et al. The complement inhibitor eculizumab in paroxysmal nocturnal hemoglobinuria. N Engl J Med 2006; 355: 1233-1243.

74 Berglund MM, Stromberg P. The clinical potential of Affibody-based inhibitors of $\mathrm{C} 5$ for therapeutic complement disruption. Expert Rev Proteomics 2016; 13: 241-243.

75 Sevigny J, Chiao P, Bussiere T, Weinreb PH, Williams L, Maier M et al. The antibody aducanumab reduces Abeta plaques in Alzheimer's disease. Nature 2016; 537: 50-56

76 Siemers ER, Sundell KL, Carlson C, Case M, Sethuraman G, Liu-Seifert H et al. Phase 3 solanezumab trials: secondary outcomes in mild Alzheimer's disease patients. Alzheimers Dement 2016; 12: 110-120.

77 Lannfelt L, Relkin NR, Siemers ER. Amyloid-ss-directed immunotherapy for Alzheimer's disease. J Intern Med 2014; 275: 284-295.

78 Hoyer W, Gronwall C, Jonsson A, Stahl S, Hard T. Stabilization of a beta-hairpin in monomeric Alzheimer's amyloid-beta peptide inhibits amyloid formation. Proc Natl Acad Sci USA 2008; 105: 5099-5104.
79 Luheshi LM, Hoyer W, de Barros TP, van Dijk Hard I, Brorsson AC, Macao B et al. Sequestration of the Abeta peptide prevents toxicity and promotes degradation in vivo. PLoS Biol 2010; 8: e1000334.

80 Lindberg H, Johansson A, Hard T, Stahl S, Lofblom J. Staphylococcal display for combinatorial protein engineering of a head-to-tail affibody dimer binding the Alzheimer amyloid-beta peptide. Biotechnol J 2013; 8: 139-145.

81 Lindberg H, Hard T, Lofblom J, Stahl S. A truncated and dimeric format of an Affibody library on bacteria enables FACS-mediated isolation of amyloid-beta aggregation inhibitors with subnanomolar affinity. Biotechnol J 2015; 10: 1707-1718.

82 Pompili M, Innamorati M, Trovarelli S, Narcisi A, Bellini S, Orsini D et al. Suicide risk and psychiatric comorbidity in patients with psoriasis. J Int Med Res 2016; 44: 61-66.

83 Gordon KB, Blauvelt A, Papp KA, Langley RG, Luger T, Ohtsuki M et al. Phase 3 trials of ixekizumab in moderate-to-severe plaque psoriasis. $N$ Engl J Med 2016; 375: 345-356.

84 Gottlieb AB, Blauvelt A, Prinz JC, Papanastasiou P, Pathan R, Nyirady J et al. Secukinumab self-administration by prefilled syringe maintains reduction of plaque psoriasis severity over 52 Weeks: results of the FEATURE trial. J Drugs Dermatol 2016; 15: 1226-1234.

85 Kontermann RE, Brinkmann U. Bispecific antibodies. Drug Discov Today 2015; 20: 838-847.

86 Kanakaraj P, Puffer BA, Yao XT, Kankanala S, Boyd E, Shah RR et al. Simultaneous targeting of TNF and Ang2 with a novel bispecific antibody enhances efficacy in an in vivo model of arthritis. MAbs 2012; 4: $600-613$

87 LaFleur DW, Abramyan D, Kanakaraj P, Smith RG, Shah RR, Wang G et al. Monoclonal antibody therapeutics with up to five specificities: functional enhancement through fusion of target-specific peptides. MAbs 2013; 5: 208-218.

88 Silacci M, Lembke W, Woods R, Attinger-Toller I, Baenziger-Tobler N, Batey $S$ et al. Discovery and characterization of COVA322, a clinical-stage bispecific TNF/IL-17A inhibitor for the treatment of inflammatory diseases. MAbs 2016; 8: 141-149.

89 Yu F, Gudmundsdotter L, Akal A, Gunneriusson E, Frejd F, Nygren PA. An affibody-adalimumab hybrid blocks combined IL- 6 and TNF-triggered serum amyloid A secretion in vivo. MAbs 2014; 6: 1598-1607.

90 Badot V, Galant C, Nzeusseu Toukap A, Theate I, Maudoux AL, Van den Eynde BJ et al. Gene expression profiling in the synovium identifies a predictive signature of absence of response to adalimumab therapy in rheumatoid arthritis. Arthritis Res Ther 2009; 11: R57.

91 Choy EH, Kavanaugh AF, Jones SA. The problem of choice: current biologic agents and future prospects in RA. Nat Rev Rheumatol 2013; 9: 154-163.

92 Ducreux J, Durez P, Galant C, Nzeusseu Toukap A, Van den Eynde B, Houssiau FA et al. Global molecular effects of tocilizumab therapy in rheumatoid arthritis synovium. Arthritis Rheumatol 2014; 66: 15-23.

93 Ogata A, Tanaka T. Tocilizumab for the treatment of rheumatoid arthritis and other systemic autoimmune diseases: current perspectives and future directions. Int J Rheumatol 2012; 2012: 946048.

94 Jones SA, Scheller J, Rose-John S. Therapeutic strategies for the clinical blockade of IL-6/gp130 signaling. J Clin Invest 2011; 121: 3375-3383.

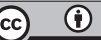

This work is licensed under a Creative Commons Attribution 4.0 International License. The images or other third party material in this article are included in the article's Creative Commons license, unless indicated otherwise in the credit line; if the material is not included under the Creative Commons license, users will need to obtain permission from the license holder to reproduce the material. To view a copy of this license, visit http:// creativecommons.org/licenses/by/4.0/ 